\title{
ROTOR RESISTANCE ESTIMATION FOR CURRENT-FED INDUCTION MOTORS
}

\author{
Dimitrios Karagiannis, Alessandro Astolfi
}

\author{
Department of Electrical and Electronic Engineering \\ Imperial College, London SW7 2BT, United Kingdom \\ Tel: +44207594 6289, Fax: +442075946282 \\ E-mail: $\{$ d.karagiannis, a.astolfi\}@imperial.ac.uk
}

\begin{abstract}
In this paper a new algorithm for estimating the rotor resistance of current-fed induction motors is presented. The proposed method does not require persistent excitation and achieves asymptotic convergence even in the case of zero rotor speed and/or low torque. However, it does require measurement of the rotor speed and flux. To make the scheme practically feasible, a method for estimating the rotor flux is devised. The proposed estimator is combined with an indirect field-oriented control law to achieve torque/speed regulation in the absence of rotor resistance and load torque information. The efficacy of the resulting adaptive output feedback control scheme is tested via simulations. Copyright (c) 2005 IFAC
\end{abstract}

Keywords: Nonlinear systems, parameter estimation, induction motors

\section{INTRODUCTION}

The problem of estimating the rotor winding resistance of induction motors has received a lot of attention recently due to its significance in improving the performance of control algorithms, as well as its application in fault detection, see e.g. (Marino et al., 1995; Marino et al., 1998; Pavlov and Zaremba, 2001) and references therein. It is well-known that the variation of the rotor resistance, which may be significant during the operation of the induction motor, can affect the performance of the controller and even lead to instability. This is particularly true for indirect field-oriented control (IFOC) schemes, which are widely considered as the industry standard, see e.g. (Leonhard, 1985; De Wit et al., 1996; Bodson and Chiasson, 1998; Peresada et al., 1999).

In this paper we develop a new method for estimating the rotor resistance of current-fed induction motors (Leonhard, 1985; Dawson et al., 1998) using measurements of the rotor speed, the elec- tromagnetic torque and the rotor flux norm. The proposed method does not require persistence of excitation and achieves asymptotic convergence even in the case of zero rotor speed and/or low torque. Moreover, it provides an asymptotic estimate of the unknown load torque. Further, the problem of torque/speed regulation by means of output feedback is addressed by combining the proposed estimator with the standard IFOC scheme.

The paper is organized as follows. Section 2 describes the dynamical model of an IFOC-driven current-fed induction motor. In Section 3 an estimator for the unknown rotor resistance and the unknown load torque is designed based on measurements of the rotor flux norm and the generated electromagnetic torque. Section 4 combines the proposed estimator with the standard IFOC to obtain an adaptive output feedback controller. Simulations of the resulting scheme are carried out in Section 5 and some conclusions are given in Section 6 . 


\section{PROBLEM FORMULATION}

The dynamical model of an induction motor in the stator reference frame (also known as $a-b$ or two-phase equivalent model) is given by the equations (Leonhard, 1985)

$$
\begin{aligned}
\dot{\lambda}_{a b}= & -\left(\frac{R_{r}}{L_{r}} I-n_{p} \omega J\right) \lambda_{a b}+\frac{M R_{r}}{L_{r}} i_{a b} \\
\dot{\omega}= & \frac{n_{p} M}{m L_{r}} i_{a b}^{T} J \lambda_{a b}-\frac{\tau_{L}}{m} \\
\dot{i}_{a b}= & \frac{M}{\sigma L_{s} L_{r}}\left(\frac{R_{r}}{L_{r}} I-n_{p} \omega J\right) \lambda_{a b} \\
& -\left(\frac{R_{s}}{\sigma L_{s}}+\frac{M^{2} R_{r}}{\sigma L_{s} L_{r}^{2}}\right) i_{a b}+\frac{1}{\sigma L_{s}} v_{a b},
\end{aligned}
$$

where $\lambda_{a b}=\left[\lambda_{a}, \lambda_{b}\right]^{T} \in \mathbb{R}^{2}$ is the rotor flux vector, $i_{a b}=\left[i_{a}, i_{b}\right]^{T} \in \mathbb{R}^{2}$ is the stator current vector, $v_{a b}=\left[v_{a}, v_{b}\right]^{T} \in \mathbb{R}^{2}$ is the stator input voltage, $\omega$ is the rotor speed, $R_{r}, L_{r}, M, n_{p}$, $m, R_{s}$ and $L_{s}$ are positive constants representing the rotor resistance, rotor inductance, mutual inductance, number of pole pairs, moment of inertia, stator resistance and stator inductance, respectively, $\sigma=1-M^{2} /\left(L_{s} L_{r}\right)$ is the leakage parameter, $\tau_{L}$ is the load torque and

$$
I=\left[\begin{array}{ll}
1 & 0 \\
0 & 1
\end{array}\right], \quad J=\left[\begin{array}{cc}
0 & -1 \\
1 & 0
\end{array}\right] .
$$

The dynamical model of the current-fed induction motor is obtained from the above equations by taking the stator current vector $i_{a b}$ as the control input, i.e. by neglecting the electromagnetic dynamics in the stator circuit. This is justified in practice by the use of high-gain current control loops. Defining the rotation matrix

$$
e^{-J n_{p} q}=\left[\begin{array}{cc}
\cos \left(n_{p} q\right) & \sin \left(n_{p} q\right) \\
-\sin \left(n_{p} q\right) & \cos \left(n_{p} q\right)
\end{array}\right],
$$

where $q$ is the rotor shaft angle, and the transformations

$$
\lambda_{r}=e^{-J n_{p} q} \lambda_{a b}, \quad i_{s}=e^{-J n_{p} q} i_{a b}
$$

yields the system

$$
\begin{gathered}
\dot{\lambda}_{r}=-\frac{R_{r}}{L_{r}} \lambda_{r}+\frac{M R_{r}}{L_{r}} i_{s} \\
\dot{\omega}=\frac{n_{p} M}{m L_{r}} i_{s}^{T} J \lambda_{r}-\frac{\tau_{L}}{m},
\end{gathered}
$$

where $\lambda_{r} \in \mathbb{R}^{2}$ is the transformed rotor flux vector and $i_{s} \in \mathbb{R}^{2}$ is the transformed stator current vector. Note that the above system describes the dynamic behavior of the current-fed induction motor in a frame rotating with angular speed $n_{p} \omega$ (Kim et al., 1997).
In the sequel, to simplify the presentation and without loss of generality, we assume that all constants are equal to one, except for the rotor resistance and the load torque which are considered unknown. Defining the control input as $u=i_{s}$ yields the simplified model

$$
\begin{gathered}
\dot{\lambda}_{r}=-R_{r} \lambda_{r}+R_{r} u \\
\dot{\omega}=u^{T} J \lambda_{r}-\tau_{L} .
\end{gathered}
$$

The indirect field-oriented controller (IFOC) is described by the equations (De Wit et al., 1996)

$$
\begin{aligned}
u & =e^{J \rho_{d}}\left[\begin{array}{c}
y_{d} \\
\tau_{d} / y_{d}
\end{array}\right] \\
\dot{\rho}_{d} & =\frac{\bar{R}_{r} \tau_{d}}{y_{d}^{2}},
\end{aligned}
$$

where $y_{d}$ and $\tau_{d}$ are the reference values of the flux norm and the torque, respectively, $\bar{R}_{r}$ is an estimate of the rotor resistance and

$$
e^{J \rho_{d}}=\left[\begin{array}{cc}
\cos \rho_{d} & -\sin \rho_{d} \\
\sin \rho_{d} & \cos \rho_{d}
\end{array}\right] .
$$

In what follows we focus (mainly) on the case of torque regulation, where $\tau_{d}$ is a constant reference, as opposed to speed regulation, where $\tau_{d}$ is the output of a PI controller, i.e.

$$
\tau_{d}=-\left(K_{P}+\frac{K_{I}}{s}\right)\left(\omega-\omega_{d}\right)
$$

where $\omega_{d}$ is the speed reference, $s$ denotes the Laplace operator and $K_{P}, K_{I}$ are constant gains. Note, however, that the two cases can be considered as approximately equivalent, if the PI is sufficiently slow.

Consider now the variables

$$
\xi_{1}=u^{T} J \lambda_{r}, \quad \xi_{2}=u^{T} \lambda_{r}, \quad y=\sqrt{\lambda_{r}^{T} \lambda_{r}}
$$

and suppose that the generated electromagnetic torque $\xi_{1}$ and the flux norm $y$ are available for measurement. The closed-loop system (5)-(7) can be rewritten in the $\xi_{1}, \xi_{2}$ and $\omega$ co-ordinates as

$$
\begin{aligned}
\dot{\xi}_{1}= & -R_{r} \xi_{1}+\dot{\rho}_{d} \xi_{2}+\frac{\dot{\tau}_{d}}{c}\left(\frac{\tau_{d}}{y_{d}^{2}} \xi_{1}+\xi_{2}\right) \\
\dot{\xi}_{2}= & -\dot{\rho}_{d} \xi_{1}-R_{r} \xi_{2}+R_{r} c \\
& +\frac{\dot{\tau}_{d}}{c}\left(-\xi_{1}+\frac{\tau_{d}}{y_{d}^{2}} \xi_{2}\right) \\
\dot{\omega}= & \xi_{1}-\tau_{L},
\end{aligned}
$$

where $c=y_{d}^{2}+\left(\tau_{d} / y_{d}\right)^{2}$, while the dynamics of the flux norm are given by

$$
\dot{y}=-R_{r} y+\frac{1}{y} R_{r} \xi_{2} .
$$


Note that, for the case of the torque regulation problem, $\dot{\tau}_{d}=0$, hence the equations (10)-(11) reduce to

$$
\begin{aligned}
& \dot{\xi}_{1}=-R_{r} \xi_{1}+\dot{\rho}_{d} \xi_{2} \\
& \dot{\xi}_{2}=-\dot{\rho}_{d} \xi_{1}-R_{r} \xi_{2}+R_{r} c .
\end{aligned}
$$

Our objective is to obtain asymptotic estimates of the rotor resistance $R_{r}$ and the load torque $\tau_{L}$ using measurements of $\xi_{1}, y$ and $\omega$.

\section{ESTIMATOR DESIGN}

Motivated by the adaptive control tools developed in (Astolfi and Ortega, 2003; Karagiannis et al., 2003) we define the error variables

$$
\begin{aligned}
& z_{1}=\hat{\tau}_{L}-\tau_{L}+\beta_{1}(\omega) \\
& z_{2}=\hat{R}_{r}-R_{r}+\beta_{2}\left(\xi_{1}\right),
\end{aligned}
$$

where $\beta_{1}(\cdot)$ and $\beta_{2}(\cdot)$ are continuous functions yet to be specified, and the update laws

$$
\begin{aligned}
\dot{\hat{\tau}}_{L}= & -\frac{\partial \beta_{1}}{\partial \omega}\left(\xi_{1}-\hat{\tau}_{L}-\beta_{1}(\omega)\right) \\
\dot{\hat{R}}_{r}= & -\frac{\partial \beta_{2}}{\partial \xi_{1}}\left[\left(-\hat{R}_{r}-\beta_{2}\left(\xi_{1}\right)\right) \xi_{1}+\dot{\rho}_{d} \xi_{2}\right. \\
& \left.+\frac{\dot{\tau}_{d}}{c}\left(\frac{\tau_{d}}{y_{d}^{2}} \xi_{1}+\xi_{2}\right)\right]
\end{aligned}
$$

where $\xi_{2}$ is obtained from the identity ${ }^{1}$

$$
\xi_{1}^{2}+\xi_{2}^{2}=c y^{2} .
$$

The resulting error dynamics are described by the equations

$$
\begin{aligned}
& \dot{z}_{1}=\frac{\partial \beta_{1}}{\partial \omega} z_{1} \\
& \dot{z}_{2}=\frac{\partial \beta_{2}}{\partial \xi_{1}} \xi_{1} z_{2} .
\end{aligned}
$$

Selecting the function $\beta_{1}(\cdot)$ as

$$
\beta_{1}(\omega)=-k_{1} \omega
$$

with $k_{1}>0$ yields the error system

$$
\dot{z}_{1}=-k_{1} z_{1}
$$

which has a globally exponentially stable equilibrium at the origin, hence $z_{1}$ converges to zero. As

\footnotetext{
1 Solving (18) for $\xi_{2}$ we obtain two solutions from which we select the positive one, i.e. $\xi_{2}=\sqrt{c y^{2}-\xi_{1}^{2}}$. This is justified by the fact that the dynamics of the square of the flux norm are given by $\dot{y}^{2}=-2 R_{r} y^{2}+2 R_{r} \xi_{2}$, hence $y^{2}$ is a filtered version of $\xi_{2}$.
}

a result, from (14) an asymptotic estimate of the load $\tau_{L}$ is given by

$$
\bar{\tau}_{L}=\hat{\tau}_{L}+\beta_{1}(\omega) .
$$

Note, moreover, that the constant $k_{1}$ which corresponds to the convergence rate of the estimation error can be arbitrarily assigned.

Consider now the problem of finding a function $\beta_{2}(\cdot)$ such that the system (20) has an asymptotically stable equilibrium at $z_{2}=0 .{ }^{2}$ A possible selection is

$$
\beta_{2}\left(\xi_{1}\right)=\frac{k_{2}}{2} \frac{1}{1+k_{3} \xi_{1}^{2}}
$$

with $k_{2}>0, k_{3}>0$ constants, yielding the stable (uniformly in $\xi_{1}$ ) error dynamics

$$
\dot{z}_{2}=-\frac{k_{2} k_{3} \xi_{1}^{2}}{\left(1+k_{3} \xi_{1}^{2}\right)^{2}} z_{2} .
$$

Remark 1. The advantage of (24) over the more obvious selection $\beta_{2}\left(\xi_{1}\right)=\frac{k_{2}}{2} \xi_{1}^{2}$ is that it ensures boundedness of $\hat{R}_{r}$ for any $\xi_{1}$. This will be particularly useful in the following section to prove stability of the adaptive closed-loop system.

Remark 2. A guideline for tuning the parameters $k_{2}$ and $k_{3}$ in (25) is to select $k_{3}$ to maximize the function $k_{3} \xi_{1}^{2} /\left(1+k_{3} \xi_{1}^{2}\right)^{2}$ for the nominal value of $\xi_{1}$ and then select $k_{2}$ according to the desired convergence rate.

From (15), assuming that $\xi_{1} /\left(1+k_{3} \xi_{1}^{2}\right)$ is not in $\mathcal{L}_{2}$, an asymptotic estimate of the rotor resistance $R_{r}$ is given by

$$
\bar{R}_{r}=\hat{R}_{r}+\beta_{2}\left(\xi_{1}\right) .
$$

Summarizing, the proposed (second-order) estimator is given by the equations (16)-(17), (21), (24) and (26).

\section{ADAPTIVE OUTPUT FEEDBACK CONTROL}

Consider again the closed-loop system (5)-(8), where $\bar{R}_{r}$ is given by (26). It was shown in the previous section that the estimate $\bar{R}_{r}$ remains bounded and asymptotically converges to the true value $R_{r}$, provided that $\xi_{1}$ is not identically equal to zero. We will now show that, for the torque regulation case, the rotor flux and the generated

\footnotetext{
2 It is interesting to note that, for any function $\beta_{2}(\cdot)$, when $\xi_{1}=0$ the system (20) has a stable equilibrium manifold given by the $z_{2}$-axis. This implies that it is not possible to estimate the rotor resistance when the torque is identically equal to zero.
} 
torque remain bounded and asymptotically converge to the reference values.

Proposition 1. Consider the IFOC-driven currentfed induction motor described by the equations (10)-(13), where $\dot{\rho}_{d}$ is given by (8) and $y_{d}$ and $\tau_{d}$ are constant references, in closed-loop with the estimator given by the equations (16)-(17), (21), (24) and

$$
\bar{R}_{r}=\max \left(\hat{R}_{r}+\beta_{2}\left(\xi_{1}\right), R_{\text {min }}\right)
$$

with $R_{\min }>0$ an arbitrarily small lower bound on $R_{r}$. Then, for all initial conditions, the states $\xi_{1}$ and $\xi_{2}$ remain bounded and, moreover,

$$
\lim _{t \rightarrow \infty} \xi_{1}(t)=\tau_{d}, \quad \lim _{t \rightarrow \infty} \xi_{2}(t)=y_{d}^{2}
$$

and

$$
\lim _{t \rightarrow \infty} y(t)=y_{d}
$$

Proof: Define the error variables

$$
x_{1}=\xi_{1}-\tau_{d}, \quad x_{2}=\xi_{2}-y_{d}^{2}
$$

and note that the system (10)-(12) can be rewritten in the $x_{1}, x_{2}$ and $\omega$ co-ordinates as

$$
\begin{aligned}
& \dot{x}_{1}=-R_{r} x_{1}+\frac{\bar{R}_{r} \tau_{d}}{y_{d}^{2}} x_{2}+\left(\bar{R}_{r}-R_{r}\right) \tau_{d} \\
& \dot{x}_{2}=-\frac{\bar{R}_{r} \tau_{d}}{y_{d}^{2}} x_{1}-R_{r} x_{2}-\left(\bar{R}_{r}-R_{r}\right) \frac{\tau_{d}^{2}}{y_{d}^{2}} \\
& \dot{\omega}=x_{1}+\tau_{d}-\tau_{L} .
\end{aligned}
$$

Consider now the Lyapunov function

$$
V\left(x_{1}, x_{2}\right)=\frac{1}{2} x_{1}^{2}+\frac{1}{2} x_{2}^{2},
$$

whose time-derivative along the trajectories of (28)-(29) satisfies

$$
\begin{aligned}
\dot{V}\left(x_{1}, x_{2}\right)= & -R_{r}\left(x_{1}^{2}+x_{2}^{2}\right) \\
& +\tau_{d}\left(\bar{R}_{r}-R_{r}\right)\left(x_{1}-\frac{\tau_{d}}{y_{d}^{2}} x_{2}\right) .
\end{aligned}
$$

A simple application of Young's inequality shows that there exist constants $\epsilon>0$ and $\delta>0$ such that

$$
\dot{V}\left(x_{1}, x_{2}\right) \leq-\epsilon V\left(x_{1}, x_{2}\right)+\delta\left(\bar{R}_{r}-R_{r}\right)^{2},
$$

hence the system (28)-(29) is input-to-state stable with respect to $\bar{R}_{r}-R_{r}$. It remains to prove that the error $\bar{R}_{r}-R_{r}$ is bounded and asymptotically converges to zero. To this end, recall first that the dynamics of the error variable $z_{2}$ defined in (15) are described by (25), hence $z_{2} \in \mathcal{L}_{\infty}$ and

$$
\frac{\xi_{1} z_{2}}{1+k_{3} \xi_{1}^{2}} \in \mathcal{L}_{2} .
$$

Since $z_{2}$ is bounded, $\bar{R}_{r}$ is also bounded, hence $\xi_{1}, \xi_{2} \in \mathcal{L}_{\infty}$ and, from (10)-(11) and (25), the time-derivatives of $\xi_{1}, \xi_{2}$ and $z_{2}$ are also bounded. From Barbalat's lemma, this implies that

$$
\lim _{t \rightarrow \infty} \frac{\xi_{1}(t) z_{2}(t)}{1+k_{3} \xi_{1}(t)^{2}}=0
$$

hence either $z_{2}$ converges to zero or $\xi_{1}$ converges to zero and $z_{2}$ converges to a nonzero constant. Due to the dynamics (10)-(11) the latter is only possible if $\dot{\rho}_{d}=0$. But from (8) and (27) we have

$$
\left|\dot{\rho}_{d}\right| \geq \frac{R_{\min }\left|\tau_{d}\right|}{y_{d}^{2}}>0
$$

Hence $z_{2}$ and therefore $\bar{R}_{r}-R_{r}$ converge to zero. As a result, $x_{1}$ and $x_{2}$ are bounded and asymptotically converge to zero, hence $\xi_{1}$ converges to $\tau_{d}$ and $\xi_{2}$ converges to $y_{d}^{2}$. This, from (13), implies that the flux norm $y$ converges to the reference value $y_{d}$.

Remark 3. A practical limitation of the proposed scheme is that it relies on measurements of the generated torque $\xi_{1}$ and the flux norm $y$, which, in turn, require knowledge of the rotor flux. To overcome this problem it is necessary to devise a method for estimating the flux vector $\lambda_{a b}$. To this end, note that from (1) and (3) we obtain

$$
\dot{\lambda}_{a b}=-\frac{\sigma L_{s} L_{r}}{M} \dot{i}_{a b}-\frac{R_{s} L_{r}}{M} i_{a b}+\frac{L_{r}}{M} v_{a b},
$$

where $\dot{i}_{a b}$ can be computed using (4) and (7). Hence, the (open-loop) observer

$$
\dot{\hat{\lambda}}_{a b}=-\frac{\sigma L_{s} L_{r}}{M} \dot{i}_{a b}-\frac{R_{s} L_{r}}{M} i_{a b}+\frac{L_{r}}{M} v_{a b}
$$

is such that $\hat{\lambda}_{a b}-\lambda_{a b}=$ const, i.e. $\hat{\lambda}_{a b}$ is an exact estimate of $\lambda_{a b}$ up to a constant error term. Since under normal operation the flux has zero mean, this error can be practically removed by filtering out the DC component of $\hat{\lambda}_{a b}$.

\section{SIMULATION RESULTS}

The model of the induction motor (1)-(3) has been simulated using the parameters $R_{r}=2$, $\tau_{L}=2$ and assuming all other constants are equal to one. A high-gain current control loop has been implemented as

$$
v_{a b}=K_{c}\left(e^{J n_{p} q} u-i_{a b}\right)
$$

with $K_{c}=500$, where $u$ is given by (7)-(8) and $\bar{R}_{r}$ is given by (27). The initial conditions are defined as $\lambda_{a b}(0)=[1,0]^{T}, \omega(0)=0$ and $i_{a b}(0)=[0,0]^{T}$. The reference of the flux norm has been set to 

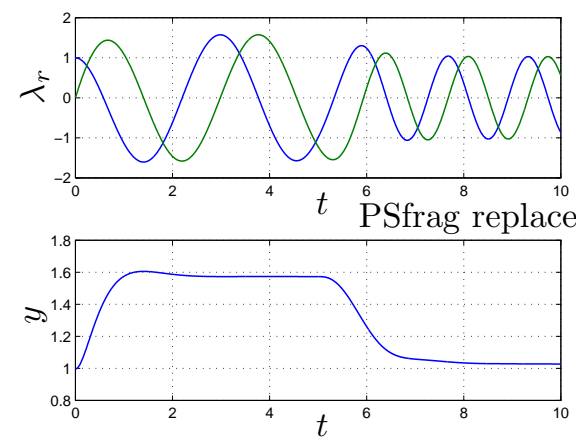

(a) Top: Rotor flux vector $\lambda_{r}$. Bottom: Flux norm $y$.
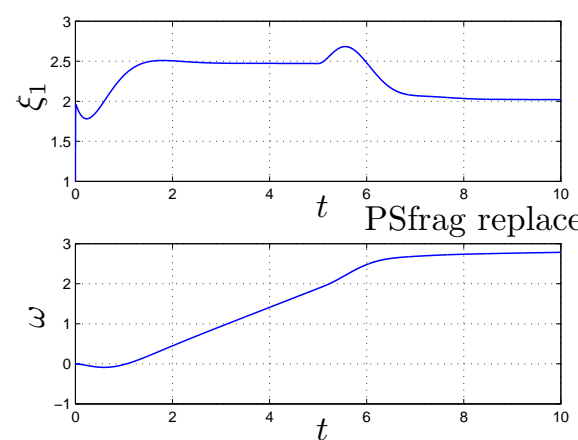

(c) Top: Generated torque $\xi_{1}$. Bottom: Rotor speed $\omega$.

Fig. 1. Time histories for the torque regulation case.

$y_{d}=1$. The adaptive gains have been set to $k_{1}=k_{2}=10$ and $k_{3}=1$. In order to compare the response with the non-adaptive case, during the first 5 s we replace $\bar{R}_{r}$ in (8) with a fixed estimate. In this case we have taken $\bar{R}_{r}=R_{r} / 2$.

\subsection{Torque regulation}

We first consider the torque regulation problem, where the torque reference is fixed at $\tau_{d}=2$. Figure 1(a) shows the time histories of the flux vector $\lambda_{r}$ and flux norm $y$, while Figure 1(b) shows the time histories of the control input $u$, the controller state $\rho_{d}$ and the flux angle. A plot of the generated torque $\xi_{1}$ is shown in Figure 1(c). Notice that, as expected from the results in (De Wit et al., 1996), the mismatch in the estimate of the rotor resistance during the first $5 \mathrm{~s}$ results in a significant steady-state error both in the flux level and in the generated torque. The convergence of the estimates $\bar{\tau}_{L}$ and $\bar{R}_{r}$ to the true values $\tau_{L}$ and $R_{r}$ is shown in Figure 1(d).

\subsection{Speed regulation}

For the speed regulation case, recall that $\tau_{d}$ is given by the PI control law (9). An implemen-


(b) Top: Stator current vector $u$. Bottom: Controller state $\rho_{d}$ (solid line) and flux angle (dashed line).
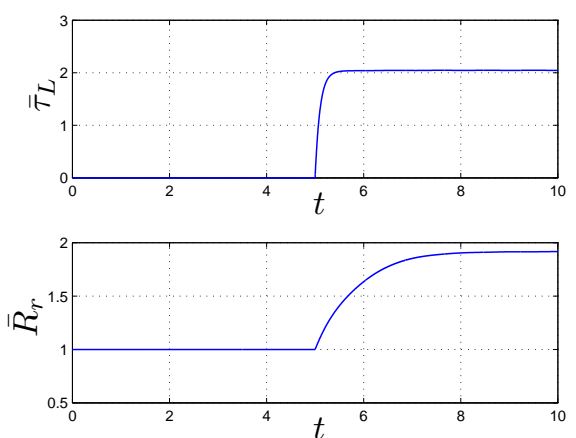

(d) Top: Load torque estimate $\bar{\tau}_{L}$. Bottom: Rotor resistance estimate $\bar{R}_{r}$.

tation of this control law, which uses the estimate of the load torque $\tau_{L}$ computed in (23), is given by

$$
\dot{\tau}_{d}=-K_{P}\left(\xi_{1}-\bar{\tau}_{L}\right)-K_{I}\left(\omega-\omega_{d}\right) .
$$

A plot of the flux vector $\lambda_{r}$ and the flux norm $y$ is shown in Figure 2(a), while Figure 2(b) shows the time histories of the control input $u$, the state $\rho_{d}$ and the flux angle. The time histories of the generated torque $\xi_{1}$ and the rotor speed $\omega$ are shown in Figure 2(c). Again we see that all signals converge to their respective reference values. Figure 2(d) shows the convergence of the estimates $\bar{\tau}_{L}$ and $\bar{R}_{r}$ to the true values $\tau_{L}$ and $R_{r}$ with a small steady-state error due to the filtering of the flux observations (see Remark 3).

\section{CONCLUSIONS}

In this paper we have presented a new algorithm for estimating the rotor resistance and load torque of a current-fed induction motor, using measurements of the rotor speed, flux magnitude and electromagnetic torque. It has been shown that the generated estimates converge asymptotically 

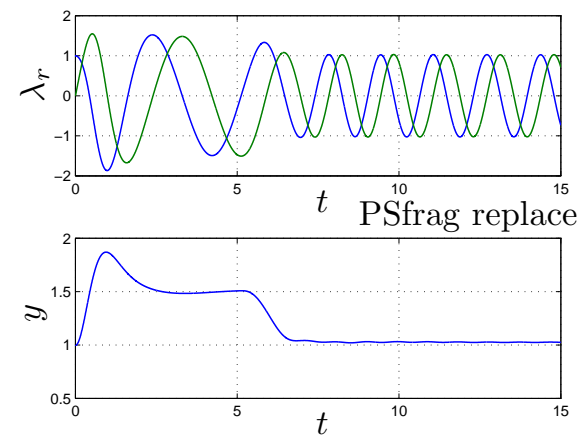

(a) Top: Rotor flux vector $\lambda_{r}$. Bottom: Flux norm $y$.
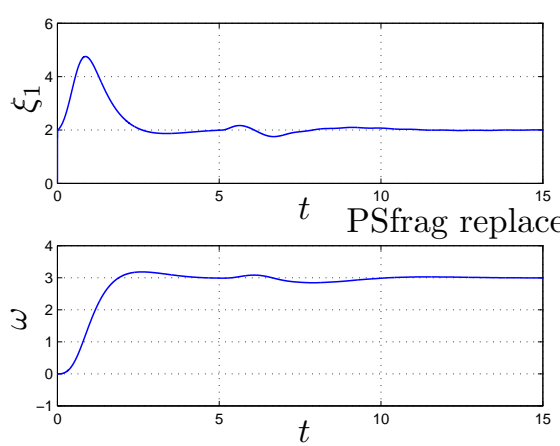

(c) Top: Generated torque $\xi_{1}$. Bottom: Rotor speed $\omega$.

Fig. 2. Time histories for the speed regulation case.

to the true values. The proposed estimator has been combined with an indirect field-oriented control law to achieve torque/speed regulation in the absence of rotor resistance or load torque information.

\section{REFERENCES}

Astolfi, A. and R. Ortega (2003). Immersion and invariance: a new tool for stabilization and adaptive control of nonlinear systems. IEEE Trans. Automatic Control 48(4), 590-606.

Bodson, M. and J. Chiasson (1998). Differentialgeometric methods for control of electric motors. Int. J. Robust and Nonlinear Control 8, 923-954.

Dawson, D.M., J. Hu and T.C. Burg (1998). Nonlinear Control of Electric Machinery. Marcel Dekker, New York.

De Wit, P., R. Ortega and I. Mareels (1996). Indirect field-oriented control of induction motors is robustly globally stable. Automatica 32(10), 1393-1402.

Karagiannis, D., A. Astolfi and R. Ortega (2003). Two results for adaptive output feedback stabilization of nonlinear systems. Automatica 39(5), 857-866.
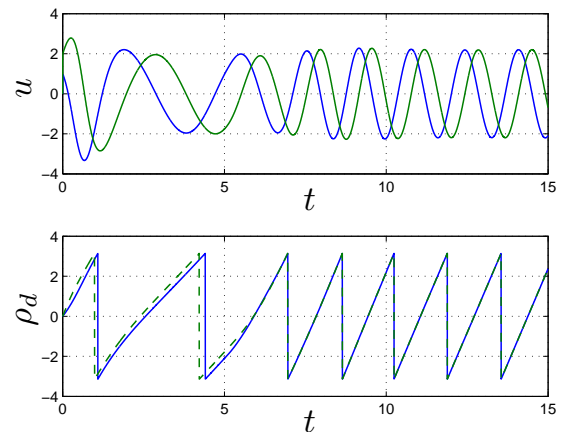

(b) Top: Stator current vector $u$. Bottom: Controller state $\rho_{d}$ (solid line) and flux angle (dashed line).
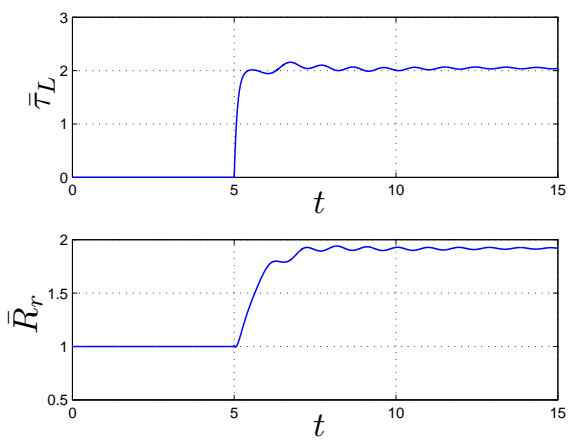

(d) Top: Load torque estimate $\bar{\tau}_{L}$. Bottom: Rotor resistance estimate $\bar{R}_{r}$.

Kim, K.-C., R. Ortega, A. Charara and J.-P. Vilain (1997). Theoretical and experimental comparison of two nonlinear controllers for current-fed induction motors. IEEE Trans. Control Systems Technology 5(3), 338-348.

Leonhard, W. (1985). Control of electrical drives. Springer-Verlag, Berlin.

Marino, R., S. Peresada and P. Tomei (1995). Exponentially convergent rotor resistance estimation for induction motors. IEEE Trans. Industrial Electronics 42(5), 508-515.

Marino, R., S. Peresada and P. Tomei (1998). Adaptive output feedback control of currentfed induction motors with uncertain rotor resistance and load torque. Automatica 34(5), 617-624.

Pavlov, A.V. and A.T. Zaremba (2001). Real-time rotor and stator resistances estimation of an induction motor. In: Proc. IFAC Symp. Nonlinear Control System Design, St. Petersburg, Russia. pp. 1252-1257.

Peresada, S., A. Tonielli and R. Morici (1999). High-performance indirect field-oriented output-feedback control of induction motors. Automatica 35(6), 1033-1047. 